\title{
Assessment of the prevalence of Streptococcus uberis in dairy cow feces and implications for herd health
}

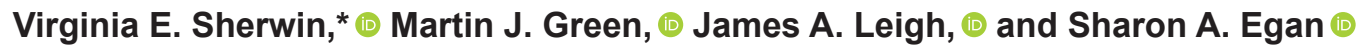 \\ School of Veterinary Science and Medicine, University of Nottingham, College Road, Sutton Bonington, Leicestershire, United Kingdom, \\ LE12 5RD
}

\begin{abstract}
Streptococcus uberis is a major causative agent of bovine mastitis worldwide, negatively affecting both milk production and animal welfare. Mammary infections result from environmental reservoirs, with cattle themselves required to propagate the infection cycle. Two longitudinal studies were performed to investigate the prevalence of Streptococcus uberis within feces and to evaluate factors which may affect gastrointestinal carriage. Bacterial detection was confirmed using a PCR-based method directed against sub0888 that detected S. uberis at an analytical sensitivity of $12 \mathrm{cfu} / \mathrm{g}$ of bovine feces. The first study sampled an entire herd at 8-wk intervals, over a 10-mo period and identified that maintenance of $S$. uberis within the dairy cow environment was due to a high proportion of animals shedding S. uberis and not due to a low number of "super-shedding" cows within the herd. Seasonality influenced detection rates, with detection levels significantly higher for housed cattle compared with those at pasture. Multilevel logistic regression was used to identify significant factors that affected S. uberis detection; these included parity, stage of lactation, and body condition score. An additional study involved screening a smaller cohort of cows housed over a 4-wk period and identified an increased probability of detection if cows were housed in loose straw yards, compared those in straw cubicles. This study highlighted several cow and management related factors that affect both detection of $S$. uberis and future infection risks.
\end{abstract}

Key words: Streptococcus uberis, dairy, mastitis, fecal detection

\section{INTRODUCTION}

Mastitis is one of the most important endemic diseases of dairy cows, resulting both in considerable eco-

Received February 16, 2021.

Accepted May 9, 2021.

*Corresponding author: Ginny.Sherwin@nottingham.ac.uk nomic loss and welfare issues for affected cows (Bradley, 2002; Halasa et al., 2007). Streptococcus uberis has been reported as the most prevalent causative agent in many countries, including the United Kingdom, with $23.5 \%$ of clinical mastitis cases attributed to S. uberis (Bradley et al., 2007; Petrovski et al., 2009; Verbeke et al., 2014). Despite concerted efforts to increase the understanding of the pathogenesis of $S$. uberis, its control has centered on treatment of infected animals and general environmental hygiene. It has been hypothesized that S. uberis resides primarily within the gastrointestinal tract of cows and is an opportunistic mammary gland pathogen, due to its asymptomatic presence at various cow sites (Cullen and Little, 1969; Lacy-Hulbert et al., 2005; Pryor, 2008) and its ability to survive in the dairy cow environment for long periods of time (Lopez-Benavides et al., 2007). The majority of $S$. uberis mastitis transmission events are hypothesized to occur through contact with environmental reservoirs in which the organism has been seeded by contamination with fecal material (Bramley et al., 1979; Zadoks et al., 2005; Lopez-Benavides et al., 2007). A few studies have identified $S$. uberis within fecal material, with prevalence rates of $5.6 \%$ in New Zealand (Lacy-Hulbert et al., 2005; Pryor, 2008) and $23 \%$ in New York state dairy herds reported (Zadoks et al., 2005). The prevalence and risk factors for S. uberis carriage and shedding within herds that are both grazed and housed has yet to be determined.

To identify S. uberis from fecal samples, several different detection methods have been employed, utilizing streptococcal specific bacterial media to enhance bacterial detection and isolate discrimination through subsequent repetitive element palindromic-PCR (Pryor, 2008) or a combination of $16 \mathrm{~S}$ rRNA PCR and ribotyping (Zadoks et al., 2005) based analysis. The recovery rates for S. uberis using culture-based methods alone has been reported to be low, with one study reporting the correct identification of S. uberis for $43 \%$ of the colonies obtained using modified Rambach media ( $\mathrm{n}=304$ out of 706 putative colonies; Zadoks et al., 2005) and may have limitations for calculating the overall prevalence 
of $S$. uberis carriage within a herd. Identification based on PCR has been used for the detection of S. uberis in mixed IMI (Gillespie et al., 1997; Ashraf et al., 2017; Soltau et al., 2017), as well as for detection of other bacteria within bovine feces, including Mycobacterium paratuberculosis (Collins et al., 1993) and Escherichia coli (Sharma, 2002). Given advances in availability of genome sequence information for $S$. uberis (Ward et al., 2009; Hossain et al., 2015), unique species-specific coding sequences have been identified and may be used in combination with culture-based detection methods to provide a specific tool for detection of S. uberis in herd prevalence studies.

Here we describe a robust method for the detection of $S$. uberis in cattle feces and its use to evaluate the herd prevalence of $S$. uberis carriage over time. This enabled investigation of factors that may affect the gastrointestinal carriage of S. uberis. By evaluating the associations between management and individual cattle factors, future risk strategies for $S$. uberis carriage and related IMI may be identified.

\section{MATERIALS AND METHODS}

\section{Fecal Sampling Strategy}

Samples were collected from a 230-dairy cow herd in Leicestershire, United Kingdom. Cattle were housed every year during winter (November to March) in a mixture of cubicles and loose straw yards and were turned out onto pasture for the rest of year. Those within 3 wk of calving were routinely housed in loose straw yards. The milking cows were fed a grass and maize silage-based partial mixed ration all year round, either with ad libitum access during housing or as a buffer feed after milking during the grazing season. Cows were fed a commercial compound concentrate in the parlor during milking. The dry cows were fed a TMR based on grass silage and straw when housed. All individual animal data (including mastitis cases) were recorded on farm databases (C21, Westfailia, GEA) and made available for this study. Somatic cell counts of individual animals were measured monthly as part of the farm's involvement in the Dairy Herd Improvement Scheme and these data were also made available for use (QMMS Ltd.).

Two separate prevalence studies were undertaken; the first evaluated patterns of shedding in all adult cows within the whole herd over 9 mo (study 1). Samples were collected approximately every 8 wk between October 2014 and June 2015. The second study (study 2) investigated patterns of shedding in a cohort of 46 cows over a consecutive 4-wk period. Animals were sampled
3 times a week during the winter housing period of February and March 2016. Body condition score was determined for all animals using a 5-point scale at each sampling time point (Edmonson et al., 1989) with each animal also scored separately for udder and leg cleanliness at each visit (Schreiner and Ruegg, 2003). Duplicate fecal samples were obtained by inserting a sterile cotton-tipped swab into the rectum or from freshly voided feces from each cow and stored at $-20^{\circ} \mathrm{C}$ until processed. Retrospective weather data were also collected from the online weather database for the weather station at Nottingham, 20 miles $(32.2 \mathrm{~km})$ from the farm (Ballester Valor, 2005).

\section{Bacterial Strains and Growth Conditions}

Unless otherwise specified, all reagents were obtained from Sigma-Aldrich. Streptococcus uberis 0140J (strain ATCC BAA-854/0140J), originally isolated from a clinical case of bovine mastitis in the United Kingdom, was used as a reference strain for this study, alongside a collection of genome-sequenced strains obtained from clinical and subclinical IMI (Hossain et al., 2015).

Bacteria were routinely cultured in Todd Hewitt broth for $16 \mathrm{~h}$ at $37^{\circ} \mathrm{C}$ unless otherwise described. The MIC of gentamicin was determined to be $4 \mu \mathrm{g} / \mathrm{mL}$ for S. uberis 0140J and was included as appropriate.

To determine detection levels of $S$. uberis, a bovine fecal sample was autoclaved at $121^{\circ} \mathrm{C}$ and confirmed to be culture negative after incubation. A culture containing $1.2 \times 10^{8} \mathrm{cfu} / \mathrm{mL}$ of $S$. uberis $0140 \mathrm{~J}$ was serially diluted and used to inoculate 1-g aliquots of feces. Seeded samples or fecal swabs were routinely cultured overnight in Todd Hewitt broth containing $4 \mu \mathrm{g} / \mathrm{mL}$ gentamicin and bacterial DNA was extracted from the culture as previously described (Leigh et al., 2010) or using the commercially available QIAamp DNA Stool Mini DNA kit (Qiagen) and used for PCR-based detection.

\section{PCR-Based Detection of S. Uberis}

Confirmation of the presence of bacterial DNA was performed by PCR amplification using primers p665 5'-AGAGTTTGATCCTGGCTCAGGA-3' and p666 5'-TTACCGCGGCTGCTGGCACGT-3', designed to amplify $16 S$ rRNA, and for $S$. uberis sub0888 primers p653 5'-CTTTATGAAAATAGCCAAGCTGAAA-3' and p654 5'-CTTAATTGTTGCTGGTCTTTCATTT-3', respectively. Approximately $10 \mathrm{ng}$ of bacterial DNA was used as a template for PCR reactions using ThemolPol Taq polymerase (New England BioLabs) according to the manufacturer's 
instructions. The DNA was separated by gel electrophoresis using $0.8 \%$ agarose $/ 40 \mathrm{~m} M$ Tris-acetate and $1 \mathrm{~m} M$ EDTA, pH 8.3 (Tris-acetate-EDTA) gel in Trisacetate-EDTA buffer for approximately $45 \mathrm{~min}$ at 100 $\mathrm{V}$ in the presence of DNA loading buffer (Bioline). Gels were stained with $0.5 \mu \mathrm{g} / \mathrm{mL}$ ethidium bromide before visualization using an UV transilluminator.

\section{Statistical and Bioinformatic Analysis}

Multilevel logistic regression models were used to evaluate the association between $S$. uberis being detected in the fecal samples (yes or no) and a series of explanatory variables. The explanatory variables tested in study 1 were parity, stage of lactation, milking status (milking or dry), BCS (and change in BCS), number of occurrences of previous S. uberis detection in that cow, high $(>200,000$ cells $/ \mathrm{mL})$ or low $(<50,000$ cells/ $\mathrm{mL}$ ) SCC, season, and type of housed environment. The explanatory variables tested in study 2 were parity, milking status (milking or dry), type of housed environment, BCS, leg and udder cleanliness score, and the number of occurrences of previous $S$. uberis detection in that cow.

The model building was conducted in MLWiN version 2.31 (Rasbash et al., 2012). Initial model building was performed by forward selection and explanatory variables were retained in the model where the estimated coefficient was greater than twice the standard error (such that the $95 \%$ CI for the estimate did not include zero, equivalent to $P<0.05$ ); all rejected variables were re-offered to the final model and retained if they now met these criteria. The model used for analysis was a 2-level hierarchical model to account for correlations between repeated samples within individual cows.

The model took the following form:

S. uberis detected (yes $=1$, no $=0) \sim \operatorname{Bernoulli}\left(\pi_{\mathrm{ij}}\right)$,

$$
\begin{gathered}
\left(\operatorname{logit}=\pi_{\mathrm{ij}}\right)=\alpha+\beta_{1} \mathrm{X}_{\mathrm{j}}+\beta_{2} \mathrm{X}_{\mathrm{ij}}+\mathrm{u}_{\mathrm{j}}, \\
{\left[\mathrm{u}_{\mathrm{j}}\right] \sim N\left(\mathbf{0}, \Omega_{\mathrm{u}}\right)}
\end{gathered}
$$

where subscripts $i$ and $j$ denoted the ith sampling point of the jth cow, respectively. $\pi_{i j}$ was the probability of detection of $S$. uberis in the feces for the ith sampling point of the jth cow, $\alpha$ was the intercept value, $X_{\mathrm{ij}}$ and $\mathrm{X}_{\mathrm{j}}$ were explanatory covariates at sampling point and cow levels, respectively, with $\beta_{\mathrm{n}}$ being the corresponding coefficients, and $u_{j}$ was the random effect to account for residual variation between cows (assumed to be normally distributed with mean $=0$ and variance $=\Omega_{\mathrm{u}}$ ).
For ease of interpretation, coefficients of the significant variables in the final models were converted to probabilities of infection and displayed graphically. Model fit was checked by evaluating and comparing model predictions with observed outcomes for different subsets of data as previously described (Gelman et al., 1996). To assess whether there was evidence of increased susceptibility of specific cows to detection of $S$. uberis in feces, an expected frequency of detection (based on the observed herd prevalence at each time point) was compared with the observed frequency.

\section{Ethics Statement}

Ethical approval for this work was granted by the Research Ethics Committee at the School of Veterinary Medicine and Science, University of Nottingham (Reference: 1750 160425).

\section{RESULTS}

\section{Optimization of PCR-Based Detection of S. Uberis}

Sub0888 primer sequence conservation was assessed using the $S$. uberis BIGSdb database (https://pubmlst .org/suberis/) containing 134 partial or completed $S$. uberis genomes. The forward primer (p653) showed $100 \%$ homology with all S. uberis strains in the database and the 25 nucleotide reverse primer could be mapped to $79 \%$ of the strains, with a maximum of 2 bp changes in the remaining $29 \%$ of strains $(15 \% 1$ mismatch, $14 \% 2$ mismatches). The primer pairs were also screened using the primer BLAST program (Ye et al., 2012) and as a sequence pair were found to only amplify the sub0888 sequence from the 4 completed S. uberis genomes within the database (accession numbers CP022435, AM946015, LS483397, and LS483408). Mismatch tolerances were investigated practically by conducting a PCR at an annealing temperature of $52^{\circ} \mathrm{C}$. This successfully amplified sub0888 from a range of clinical strains including EF20, B362, and 6780, which had 1 (B362 and 6780) or 2 (EF20) nucleotide mismatches in the reverse primer sequences (Figure 1; Hossain et al., 2015).

Cattle fecal aliquots spiked with serially diluted S. uberis 0140J were processed to extract DNA from enrichment cultures. The presence of bacterial DNA was confirmed by $16 S$ rDNA-based PCR (Figure 2A and $\mathrm{C}$ ) and presence of $S$. uberis by PCR amplification of sub0888 (Figure 2B and D). Streptococcus uberis DNA was be detected in fecal samples seeded with as few as $12 \mathrm{cfu}$ using the method developed by Leigh et al. (2010). The QIAamp DNA Stool Mini DNA kit failed to extract sufficient $S$. uberis DNA for analysis. 


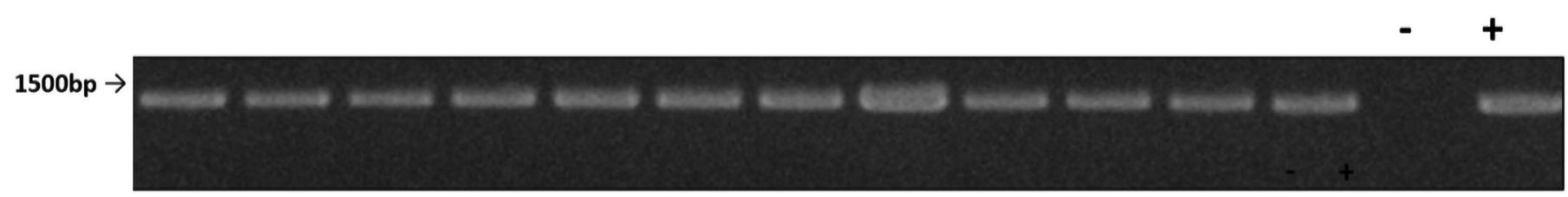

Figure 1. Detection of sub0888 within 12 field isolates of Streptococcus uberis. (-) no template DNA, (+) 0140J control.

\section{Study 1: A Longitudinal Study Investigating the Herd Prevalence of S. Uberis in Bovine Feces}

As part of study 1 , fecal samples were taken every $8 \mathrm{wk}$, resulting in a mean of 216 cows sampled per time point and a total of 1,080 samples collected over a period of $32 \mathrm{wk}$.

The overall prevalence of $S$. uberis positive fecal samples was 195 out of 1,080 samples $(18 \%)$ with increased detection in samples collected during winter months of December and February, when animals were housed rather than at pasture (Table 1). A total of 133 cows were tested on all 5 sampling occasions with $34 \%$ $(\mathrm{n}=45)$ that did not test positive for $S$. uberis at any time point. No cows tested positive on all 5 occasions, whereas $66 \%(\mathrm{n}=88)$ were positive for $S$. uberis on at least one occasion (Figure $3 \mathrm{~A}$ ). To assess whether there was evidence of increased detection of $S$. uberis in feces of specific cows, an expected frequency of detection (based on the observed herd prevalence at each time point) was compared with the observed frequency. The proportion of cows identified as shedding on more than one occasion aligned with that expected if the probability of shedding at each time point was equal for all cows (i.e., a random process). Although S. uberis was detected in some cows more frequently than others, this was no more than would have been expected if the process of shedding was a random event across all cows.

The results of the final multilevel logistic regression model are presented in Table 2. Significant variables affecting the detection of S. uberis were BCS, parity, DIM, and housing conditions. The odds of detecting
S. uberis in fecal samples of cows increased by 2.8 times during the winter-housed period compared with the grazing period. There was an increased odds of $S$. uberis detection if the cows were in early lactation (0-49 DIM) compared with later on during lactation $(\geq 49$ DIM) or when dry, as well as if they had a BCS $\geq 3.5$ compared with $<3.5$ or were in their first lactation compared with multiparous cows. Model predictions and observed outcomes indicated that the model fit was good. The significant associations identified in the model are illustrated graphically in Figures 4 and 5.

\section{Study 2: A Cohort Study Investigating the Prevalence of S. Uberis in Bovine Feces}

The mean prevalence of detection during the cohort study, which was conducted over a 4 -wk period in February 2016, was $30 \%$ (Table 3 ). This was similar to the mean prevalence of $33 \%$ identified in study 1 for the February sampling time point. The prevalence varied considerably over the duration of study 2 (from 13.0 to $54.3 \%$ ). Fifteen percent of the cohort were positive on more than $50 \%$ of sampling occasions and no cows tested positive on more than $75 \%$ of sampling occasions in the 4 -wk sampling period. Four out of the 46 cows did not test positive for $S$. uberis at any time point (Figure 3B).

The weather recordings taken from the local weather station were analyzed to investigate whether the temperature or humidity of the day was associated with the fecal detection of S. uberis (Table 3). There was no apparent association between the detection of $S$. uberis

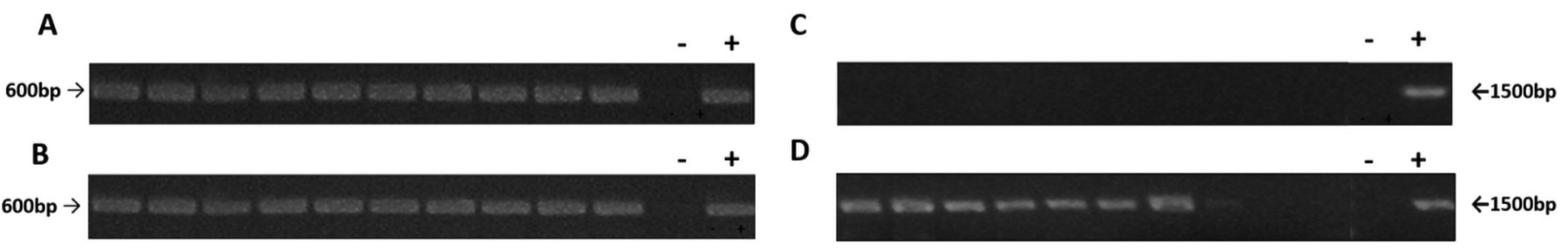

Figure 2. Streptococcus uberis detection by PCR using different DNA extraction techniques. Autoclaved fecal samples inoculated in brain heart infusion containing $4 \mu \mathrm{g} / \mathrm{mL}$ gentamicin were spiked with serial dilutions of $S$. uberis $0104 \mathrm{~J}$ beginning at $1.2 \times 10^{8}$ cfu/sample. The samples were cultured overnight at $37^{\circ} \mathrm{C}$. The DNA was extracted using either the QIAamp DNA stool mini kit (A and C; Qiagen) or phenol method (B and D). The presence of bacterial DNA was determined using 16S PCR (A and B). The presence of $S$. uberis DNA was determined using sub0888 PCR (B and D). (-) no template DNA, (+) 0140J control. 
Table 1. Prevalence of Streptococcus uberis in fecal samples over a 10-mo period in a UK dairy herd

\begin{tabular}{lccccc}
\hline Item & Oct 2014 & Dec 2014 & Feb 2015 & Apr 2015 & Jun 2015 \\
\hline Cow environment & Pasture & Housed & Housed & Pasture & Pasture \\
Number of cows tested & 226 & 209 & 202 & 219 & 224 \\
Percentage of herd testing positive & 10.2 & 25.4 & 33.2 & 12.3 & 11.2 \\
\hline
\end{tabular}

A

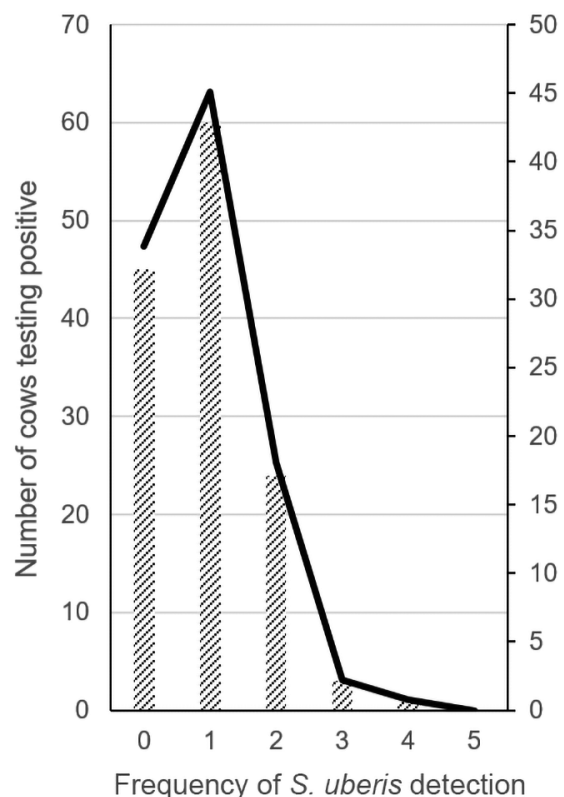

B

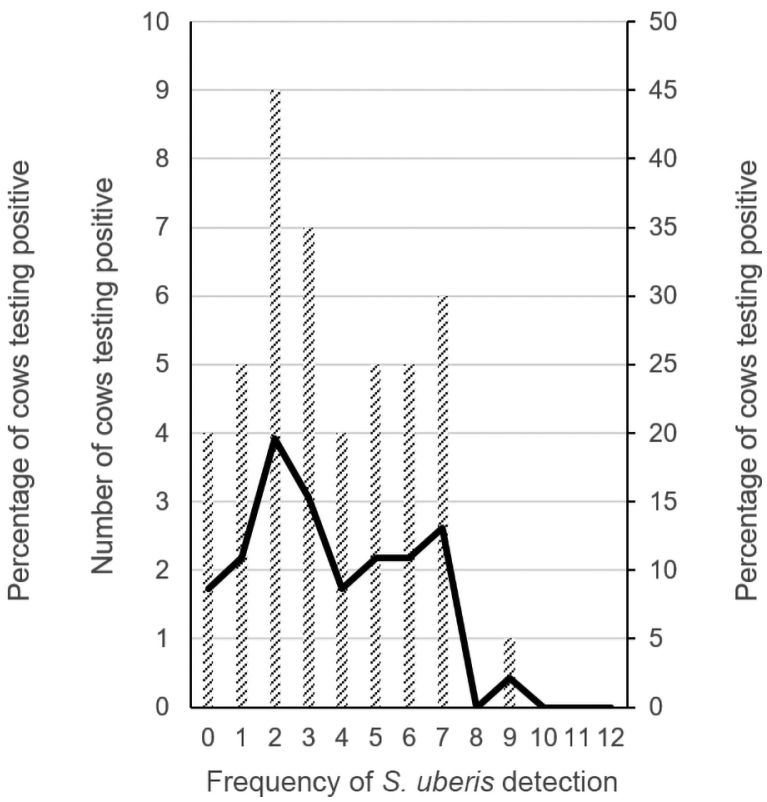

Figure 3. Frequency of Streptococcus uberis detection in fecal samples within an individual cow over the sample periods. (A) Herd prevalence for study 1 ; cows sampled on 5 occasions over 32 -wk period $(n=133)$. (B) Cohort prevalence for study 2 ; cows sampled on 12 occasions over 4 -wk period $(\mathrm{n}=46)$.

Table 2. Parameter estimates from the final multilevel logistic regression model for detection of Streptococcus uberis in feces when investigating whole-herd prevalence

\begin{tabular}{lcccc}
\hline Model term $^{1}$ & Number of samples & Odds ratio & CI & $P$-value \\
\hline $\begin{array}{l}\text { Outdoor season } \\
\text { (Oct, Apr, and Jun) }\end{array}$ & 669 & Referent & Referent & \\
$\begin{array}{l}\text { Inside season } \\
\text { (Dec and Feb) }\end{array}$ & & & & \\
0-49 DIM & 411 & 2.82 & $1.95-4.07$ & $<0.05$ \\
50-99 DIM & 151 & Referent & Referent & \\
100-149 DIM & 111 & 0.30 & $0.16-0.59$ & $<0.05$ \\
150-200 DIM & 145 & 0.57 & $0.33-0.99$ & $<0.05$ \\
200-249 DIM & 135 & 0.42 & $0.24-0.75$ & $<0.05$ \\
$\geq 250$ DIM & 126 & 0.28 & $0.14-0.53$ & $<0.05$ \\
Dry cows & 148 & 0.33 & $0.18-0.58$ & $<0.05$ \\
BCS $\leq 2.0$ & 164 & 0.29 & $0.15-0.56$ & $<0.05$ \\
BCS 2.5 & 145 & 0.56 & $0.33-0.99$ & $<0.05$ \\
BCS 3.0 & 281 & 0.54 & $0.34-0.86$ & $<0.05$ \\
BCS $\geq 3.5$ & 295 & 0.59 & $0.37-0.94$ & $<0.05$ \\
Parity 1 & 226 & Referent & Referent & $<0.05$ \\
Parity 2 and above & 268 & Referent & Referent & $<0.05$ \\
\hline
\end{tabular}

${ }^{1}$ Nonsignificant factors omitted included change in BCS, milking status (dry or milking), SCC, previous and future fecal detection of S. uberis, and housing (cubicles or straw yards). 


\section{- - - Average cow outdoors Average cow indoors}

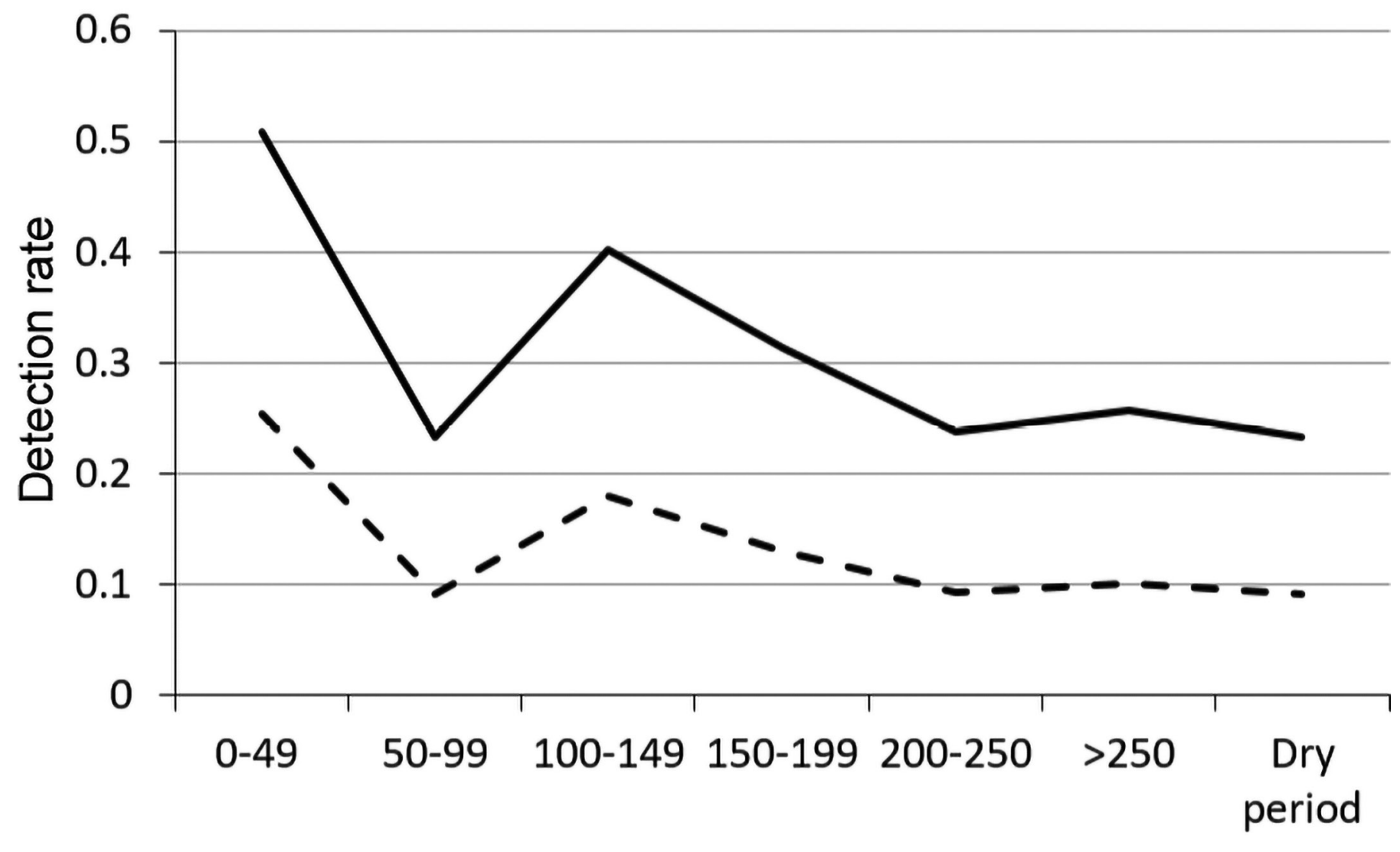

\section{Stage of lactation (DIM)}

Figure 4. Predicted probability and observed prevalence of Streptococcus uberis detection over the lactation period. Graphical representation of the predicted risk of detecting S. uberis in the cattle feces when grazing (dotted line) or housed (solid line) over the course of the lactation period (x-axis).

and changes in weather conditions, such as increased rainfall, humidity, or abnormal temperature fluctuations. In addition, there were no changes in yard management, such as frequency of cleaning of straw yards or dietary alterations that accompanied the changes in prevalence. Individual cow factors such as cleanliness scoring were also not significantly different for these time points and no other factors were correlated with these fluctuations.

The results of the final multilevel model for study 2 (Table 4) did not substantiate that the variables BCS, DIM, and parity were significant covariates, but did indicate that the odds of detection increased if the cows were housed in loose straw yards compared with straw cubicle yards and if the cow had tested positive on least one occasion, compared with no S. uberis detection, in the previous 3 tests.

\section{DISCUSSION}

The key to reducing environmental sources of mastitis within a herd is to reduce the exposure of cows to pathogens such as S. uberis (Hogan and Smith, 2012). Fecal material is thought to provide a continuous reservoir for $S$. uberis by seeding the dairy cow environment (Zadoks et al., 2005; Lopez-Benavides et al., 2007). Understanding the effect of environmental and management changes or cattle factors that increase $S$. uberis prevalence in feces may play a key role in controlling the level of environmental contamination and subsequent risks for IMI.

The method developed for the identification of $S$. uberis in this study used a combination of bacterial enrichment by culture- and species-specific PCR, which proved to be a simple and robust method for detec- 

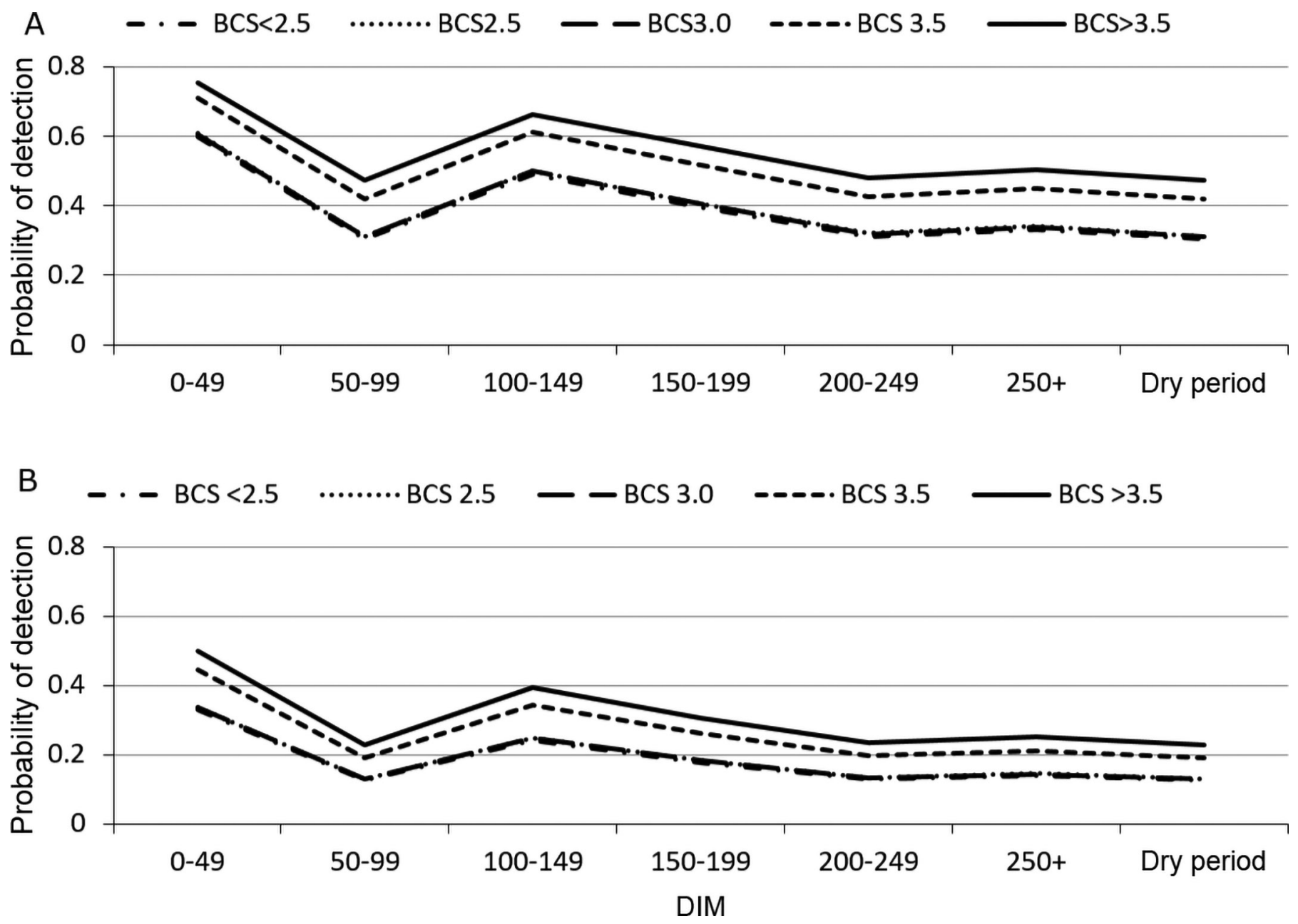

Figure 5. (A and B) The probability of detection of Streptococcus uberis in feces during the housing period at different BCS or lactation time points. The combined effects of the significant variables from the logistic regression model were transformed into the risks of the probability of detection of $S$. uberis in the feces of dairy cows and presented in graphical format. The x-axis indicates the stage of lactation, in terms of DIM. Each of the lines indicates the BCS of the first-lactation heifers, scored on a scale of 1 to 5 , based on the Agriculture and Horticulture Development Board scoring system. This indicates an increased probability of detection of S. uberis in heifers that had an increased BCS and were early in lactation. (A) Represents first-lactation heifers during the housing period, with the probability of detection of detection of $S$. uberis in their feces of 0.753 in the first $50 \mathrm{~d}$ of lactation if the BCS is $>3.5$, compared with a probability of detection of 0.608 if the BCS is 2.5. (B) Represents multiparous cows during the housing period, with the probability of detection of detection of $S$. uberis in their feces of 0.672 in the first $50 \mathrm{~d}$ of lactation if the BCS is $>3.5$, compared with a probability of detection of 0.510 if the BCS is 2.5 .

tion of as few as 12 cfu of $S$. uberis within the mixed bacterial populations in fecal samples (Figure 2D). The target selected for detection, sub0888 (SUB_RS04480), encodes a sortase anchored protein of unknown function (Egan et al., 2010; Leigh et al., 2010) and was shown to be present in all S. uberis genome sequences within the National Center for Biotechnology Information RefSeq database, as well as those partial sequences within the S. uberis BIGSdb database (Altschul et al., 1990; Jolley and Maiden, 2010); sub0888 is considered unique to S. uberis (Ward et al., 2009). Amplification of this sequence by PCR was shown to tolerate 1 to 2 nucleotide mismatches within the primer sequences.
The overall prevalence of $S$. uberis positive samples for the herd analyzed in study 1 was $18.1 \%$ ( $\mathrm{n}=195$ positive samples out of 1,080 ), with a wide variation in detection rates of $S$. uberis, ranging from $10.2 \%$ during the grazing season (October 2014) to $33.2 \%$ during the housed season (February 2015). Similar detection rates for $S$. uberis over the housing period were observed in both study 1 and study $2(29.3 \%$ and $30.1 \%$, respectively), suggesting a consistent rate of detection of S. uberis under these conditions. The detection levels during the grazing period of study 1 were similar to the prevalence of S. uberis reported from a grazing herd in New York, over a 4-mo period (Zadoks et al., 2005); however, in 


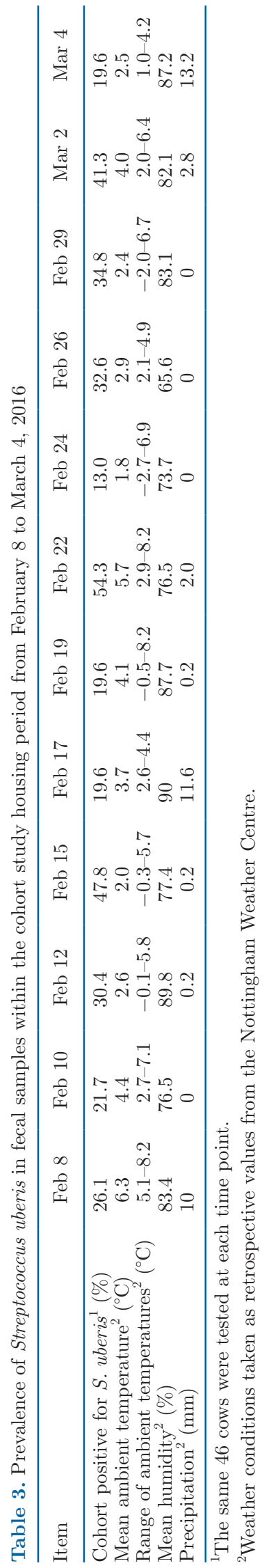

the reported study, sampling was only conducted on cows before and after calving and not on all animals within the herd. In contrast, the mean detection level in study 1 was 3 times higher than that reported in a study of grazing dairy cows in New Zealand over a 15 -mo period, which was found to be between $1.2 \%$ to 9.7\% (Pryor, 2008). However, the prevalence of detection in the New Zealand pasture only herd was similar to prevalence of detection in our study herd when the cows were on an outdoor diet consisting of pasture, partial mixed ration, and concentrate feeding $(7.9 \%, \mathrm{n}$ $=53$ out of 669), which suggests that the effect of diet on detection of $S$. uberis could be limited, or that there are multiple interacting factors, of which diet is only one. Direct comparisons between these studies are difficult due to differences in study design, management systems, S. uberis detection methods, and potential breed differences alongside influence from geographical or climate factors.

In study 1 , there was an increased odds of $S$. uberis detection in the winter months (odds ratio 2.82), which may reflect changes in cow management, including stocking density and differences in the diet, as well as changes in cattle behavior and local environment that could directly affect the efficacy of S. uberis feco-oral transmission. A significant seasonal trend in the detection of $S$. uberis from different sites on and within the cow has been reported previously (Buddle et al., 1988). This coincided with an increased detection rate of S. uberis during the winter months, in comparison to spring and autumn, which mirrored the increased detection seen over similar time points in the present study. Previous research has shown that cattle do not preferentially defecate in particular areas while grazing (Oudshoorn et al., 2008), but do have specific preferential areas within cubicle housing (Villettaz Robichaud et al., 2011). This potentially could lead to a higher concentration of $S$. uberis in particular sites within housing and therefore increase the risk of feco-oral transmission and transmission to the mammary gland.

Alteration to the diet is a potential external factor that could influence $S$. uberis presence and detection within feces and differed between the seasons in study 1 , with the inclusion of grazed pasture in the summer season. Analysis of the S. uberis 0140J genome shows a diverse collection of genes indicative of the ability to utilize a range of different carbohydrates as a source of carbon and energy (Ward et al., 2009), which may favor survival or proliferation in certain gastrointestinal conditions. Another hypothesis is that differences in diet may alter fecal consistency, providing a potential dilution effect within the feces when the cows are at pasture and resulting in a decreased chance of $S$. uberis detection. The effect of diet on fecal shedding of $S$. 
uberis is currently unknown and further research in this area is required; the protocols developed in this study would greatly facilitate such investigation.

Other management factors were highlighted to have an effect on the detection of $S$. uberis in feces, with increased odds of detection of S. uberis if cattle were housed in loose straw yards compared with those in straw cubicles (odds ratio: 1.77). Although this could be related to differences in management of the housing barns, it may also be influenced by factors such as humidity, $\mathrm{pH}$, and temperature of the bedding, which have previously been shown to influence both the growth rate and survival of S. uberis (Ward et al., 2002). This suggests that barn design, environmental conditions, bedding substrate, and overall cleanliness may affect the density of $S$. uberis within the environment and should be considered as possible methods for reducing the levels of $S$. uberis within the environment by limiting the feco-oral transmission.

The presence of persistent shedding cattle acting as reservoirs of infection with $S$. uberis within cattle herds has been debated (Lacy-Hulbert et al., 2005; Zadoks et al., 2005). In study 1 , there was little evidence that individual cattle within the herd could be identified as persistent shedders of $S$. uberis over a long period of time, with no cows identified as positive for $S$. uberis in fecal sampling at every time point and therefore detection of $S$. uberis was random in terms of long-term detection (Figures 3 and 4). However, in the short-term study, the previous detection of $S$. uberis increased the odds of detecting $S$. uberis within the feces again within the next $6 \mathrm{~d}$ (odds ratio: 2.28). These data suggest that long-term gastrointestinal colonization is unlikely, but are consistent with a model in which $S$. uberis is a transient but frequent part of the gastrointestinal flora that requires repopulation by repeated ingestion. Clearly the ruminant gut represents a complex ecosystem and further research is required to determine factors (bacterial host and environment) that underpin frequency and duration carriage of $S$. uberis and the potential effect this has on IMI.

The effect of individual cow factors was found to differ between the long-term (study 1) and short-term (study 2 ) studies in terms of their effects on the probability of detection of $S$. uberis in feces. The difference between the 2 studies reflects the fact that study 2 was not designed to detect effects of individual cow factors and therefore sample size and analytic power were not comparable between studies. Stage of lactation was shown to be significant in study 1 , with early-lactation cows having an increased odds of $S$. uberis detection. This differed from findings in a New Zealand study, which highlighted a peak in S. uberis detection in grazing dry cows in a spring block calving herd (Lacy-Hulbert et al., 2005). These 2 studies suggest that changes around drying off and calving may influence the dynamics of gastrointestinal microbiome, which could be related to alterations in management factors such as diet, or alterations in the physiology of the cow. However another study showed no significant difference when comparing fecal shedding at dry off and calving periods (Zadoks et al., 2005). These studies suggest that any differences due to stage of lactation may have confounding issues relating to animal management around calving and in early lactation and require further investigation.

The effects of parity and BCS on the prevalence of fecal shedding has not previously been reported. The importance of BCS may reflect differences in underlying physiology, which could correlate to potential differences in gastrointestinal tract conditions. Parity may reflect increased colonization of first-lactation heifers, which are exposed to a different environment upon entering the herd, or potentially reflects a difference in the physiological or immunological status of the animal, in comparison to multiparous animals. In this instance, the effects of parity and BCS are unlikely to reflect the effect of diet, because all animals had access to the same diet at each time point. Because this is the first report of significant cow factors affecting the detection of $S$. uberis, further research is required to understand the clinical importance.

There were limitations to both of the studies, including the inability to generalize the findings to other dairy farms, as the studies were conducted on one herd in the United Kingdom and therefore would need to be repeated on different farms with different management and housing systems to understand farm- and cow-related variation. Limitations in study 1 included

Table 4. Parameter estimates from the final multilevel logistic regression model for Streptococcus uberis detection in feces in study 2

\begin{tabular}{lccc}
\hline Model term $^{1}$ & Number of samples & Odds ratio & CI \\
\hline Cubicle housing & 326 & Referent & Referent \\
Straw housing & 226 & 1.77 & $1.13-2.76$ \\
S. uberis not detected in previous 3 tests & 159 & Referent & Referent \\
S. uberis detected at least once in previous 3 tests & 255 & 2.28 & $1.37-3.77$ \\
\hline
\end{tabular}

${ }^{1}$ Nonsignificant variables that were also tested included parity, milking status (dry or milking), BCS, leg cleanliness, udder cleanliness, and detection of $S$. uberis in the previous immediate test or previous 2 tests or if the cow had ever tested positive at previous time points. 
the inability to sample all the same cows at each time point, due to the logistics of using a commercial dairy farm. The potential for selection bias through weighted sampling of certain groups within the population was avoided by sampling all cows present on the day in study 1 and by ensuring a representative range of parities and stages of lactation within the cohort in study 2. In both studies, only one fecal swab was processed (due to limited resource availability) and therefore accuracy of results relied on the assumption that S. uberis was homogeneously spread throughout feces. Mixing of ingesta and feces is likely to be thorough due to ruminal contractions and intestinal peristalsis, suggesting homogeneous spreading of bacteria throughout a fecal stool is not an unreasonable assumption. It is possible that the detection rates reported in this research are underestimates of the true prevalence; however, the results indicate that the sampling methodology and laboratory testing were sufficiently accurate to detect significant associations between explanatory variables despite any measurement error that may have been associated with the detection of $S$ uberis in feces.

\section{CONCLUSIONS}

The results of this study highlight the potential effects of both management and cow factors on the detection of $S$. uberis in bovine feces. As such, these factors will play a role in the persistence and transmission of S. uberis within the dairy herd environment. The development of a simple and robust molecular tool for the detection of $S$. uberis within the feces of cows allowed the use of a repeatable screening test with a high analytical sensitivity for an epidemiological study. It was observed in both studies that $S$. uberis could be detected in the feces of the majority of cows within the studied population. The seasonal increase in fecal detection of $S$. uberis detected in study 1 corresponded with an increased incidence of clinical mastitis during the housed winter period. By extension, this suggests there are factors in the management of the dairy cow population that influence infectious pressure of $S$. uberis by affecting the level of $S$. uberis in the environment by virtue of gut carriage and fecal shedding. The tool developed here will permit further investigation to understand (and reduce) environmental seeding by $S$. uberis, which in turn could reduce the infection pressure for IMI with this recalcitrant pathogen.

\section{ACKNOWLEDGMENTS}

This research was carried out as part of a Streptococcus uberis research project funded by the Barham Benevolent Foundation (Chichester, UK) and the Uni- versity of Nottingham School of Veterinary Medicine and Science (Leicestershire, UK). The authors have not stated any conflicts of interest. V.S., S.E., J.L., and M.G. conceived and planned the study. V.S., S.E., and J.L. developed the laboratory diagnostic test to detect S. uberis in bovine feces. V.S. collected the samples and analyzed the fecal samples. V.S. and M.G. performed the statistical analysis, with V.S., M.G., J.L., and S.E. contributing the interpretation of the results. V.S. took the lead in writing the manuscript, with all authors providing critical feedback and helping shape the research, analysis, and manuscript.

\section{REFERENCES}

Altschul, S. F., W. Gish, W. Miller, E. W. Myers, and D. J. Lipman. 1990. Basic local alignment search tool. J. Mol. Biol. 215:403-410. https://doi.org/10.1016/S0022-2836(05)80360-2.

Ashraf, A., M. Imran, T. Yaqub, M. Tayyab, W. Shehzad, and P. Thomson. 2017. A novel multiplex PCR assay for simultaneous detection of nine clinically significant bacterial pathogens associated with bovine mastitis. Mol. Cell. Probes 33:57-64. https://doi .org/10.1016/j.mcp.2017.03.004.

Ballester Valor, G. 2005. Ogimet [Online]. Accessed May 24, 2020 https://www.ogimet.com/bufr.phtml.en.

Bradley, A. 2002. Bovine mastitis: An evolving disease. Vet. J. 164:116-128. https://doi.org/10.1053/tvjl.2002.0724.

Bradley, A. J., K. A. Leach, J. E. Breen, L. E. Green, and M. J. Green. 2007. Survey of the incidence and aetiology of mastitis on dairy farms in England and Wales. Vet. Rec. 160:253-258. https://doi .org/10.1136/vr.160.8.253.

Bramley, A. J., M. F. H. Shearn, and R. G. Kingwell. 1979. The isolation of Streptococcus uberis from cattle bedding and faeces. Pages 142-143 in XX International Dairy Congress, Paris, France.

Buddle, B. M., J. R. Tagg, and M. J. Ralston. 1988. Use of an inhibitor typing scheme to study the epidemiology of Streptococcus uberis mastitis. N. Z. Vet. J. 36:115-119. https://doi.org/10.1080/ 00480169.1988.35504.

Collins, D. M., D. M. Stephens, and G. W. De Lisle. 1993. Comparison of polymerase chain reaction tests and faecal culture for detecting Mycobacterium paratuberculosis in bovine faeces. Vet. Microbiol. 36:289-299. https://doi.org/10.1016/0378-1135(93)90095-O.

Cullen, G. A., and T. W. A. Little. 1969. Isolation of Streptococcus uberis from the rumen of cows and from soil. Vet. Rec. 85:115-118. https://doi.org/10.1136/vr.85.5.115.

Edmonson, A. J., I. J. Lean, L. D. Weaver, and T. Farver. 1989. A body condition scoring chart for Holstein dairy cow. J. Dairy Sci. 72:68-78. https://doi.org/10.3168/jds.S0022-0302(89)79081-0.

Egan, S. A., D. Kurian, P. N. Ward, L. Hunt, and J. A. Leigh. 2010. Identification of sortase A (SrtA) substrates in Streptococcus uberis: Evidence for an additional hexapeptide (LPXXXD) sorting motif. J. Proteome Res. 9:1088-1095. https://doi.org/10.1021/ pr901025w.

Gelman, A., X. Meng, and H. Stern. 1996. Posterior predictive assessment of model fitness via realized discrepancies. Stat. Sin. 6:733-760.

Gillespie, B. E., B. M. Jayarao, and S. P. Oliver. 1997. Identification of Streptococcus species by randomly amplified polymorphic deoxyribonucleic acid fingerprinting. J. Dairy Sci. 80:471-476. https://doi .org/10.3168/jds.S0022-0302(97)75959-9.

Halasa, T., K. Huijps, O. Osteras, and H. Hogeveen. 2007. Economic effects of bovine mastitis and mastitis management: A review. Vet. Q. 29:18-31. https://doi.org/10.1080/01652176.2007.9695224.

Hogan, J.., and L. Smith. 2012. Managing environmental mastitis. Vet. Clin. North Am. Food Anim. Pract. 28:217-224. https://doi.org/ 10.1016/j.cvfa.2012.03.009. 
Hossain, M., S. A. Egan, T. Coffey, P. N. Ward, R. Wilson, J. A. Leigh, and R. D. Emes. 2015. Virulence related sequences; insights provided by comparative genomics of Streptococcus uberis of differing virulence. BMC Genomics 16:334. https://doi.org/10.1186/ s12864-015-1512-6.

Jolley, K. A., and M. C. Maiden. 2010. BIGSdb: Scalable analysis of bacterial genome variation at the population level. BMC Bioinformatics 11:595. https://doi.org/10.1186/1471-2105-11-595.

Lacy-Hulbert, S. J., R. T. Cursons, M. G. Lopez-Benavides, J. H. Williamson, E. L. Summers, S. M. Pryor, and W. M. Woolford. 2005. Isolation of Streptococcus uberis from different sites of the dairy cow. In Mastitis in Dairy Production: Current Knowledge and Future Solutions. H. Hogeveen, ed. Wageningen Academic Publishers.

Leigh, J. A., S. A. Egan, P. N. Ward, T. R. Field, and T. J. Coffey. 2010. Sortase anchored proteins of Streptococcus uberis play major roles in the pathogenesis of bovine mastitis in dairy cattle. Vet. Res. 41:63. https://doi.org/10.1051/vetres/2010036.

Lopez-Benavides, M. G., J. H. Williamson, G. D. Pullinger, S. J. LacyHulbert, R. T. Cursons, and J. A. Leigh. 2007. Field observations on the variation of Streptococcus uberis populations in a pasturebased dairy farm. J. Dairy Sci. 90:5558-5566. https://doi.org/10 .3168/jds.2007-0194.

Oudshoorn, F. W., T. Kristensen, and E. S. Nadimi. 2008. Dairy cow defecation and urination frequency and spatial distribution in relation to time-limited grazing. Livest. Sci. 113:62-73. https://doi .org/10.1016/j.livsci.2007.02.021.

Petrovski, K. R., C. Heuer, T. J. Parkinson, and N. B. Williamson. 2009. The incidence and aetiology of clinical bovine mastitis on 14 farms in Northland, New Zealand. N. Z. Vet. J. 57:109-115. https: //doi.org/10.1080/00480169.2009.36887.

Pryor, S. M. 2008. Bovine mastitis and ecology of Streptococcus uberis. $\mathrm{PhD}$ Thesis, The University of Waikato, Hamilton, New Zealand.

Rasbash, J., C. Charlton, W. J. Browne, M. Healy, and B. Cameron. 2012. MLwiN Version 2.10. Centre for Multilevel Modelling, University of Bristol.

Schreiner, D. A., and P. L. Ruegg. 2003. Relationship between udder and leg hygiene scores and subclinical mastitis. J. Dairy Sci. 86:3460-3465. https://doi.org/10.3168/jds.S0022-0302(03)73950 -2 .

Sharma, V. K. 2002. Detection and quantitation of enterohemorrhagic Escherichia coli O157, O111, and O26 in beef and bovine feces by real-time polymerase chain reaction. J. Food Prot. 65:1371-1380. https://doi.org/10.4315/0362-028X-65.9.1371.
Soltau, J., E. Einax, K. Klengel, J. Katholm, K. Failing, A. Wehrend, and K. Donat. 2017. Within-herd prevalence thresholds for herd-level detection of mastitis pathogens using multiplex realtime PCR in bulk tank milk samples. J. Dairy Sci. 100:8287-8295. https://doi.org/10.3168/jds.2016-12385.

Verbeke, J., S. Piepers, K. Supre, and S. De Vliegher. 2014. Pathogenspecific incidence rate of clinical mastitis in Flemish dairy herds, severity, and association with herd hygiene. J. Dairy Sci. 97:69266934. https://doi.org/10.3168/jds.2014-8173.

Villettaz Robichaud, M., A. M. de Passillé, D. Pellerin, and J. Rushen. 2011. When and where do dairy cows defecate and urinate? J. Dairy Sci. 94:4889-4896. https://doi.org/10.3168/jds.2010-4028.

Ward, P. N., M. T. Holden, J. A. Leigh, N. Lennard, A. Bignell, A. Barron, L. Clark, M. A. Quail, J. Woodward, B. G. Barrell, S. A. Egan, T. R. Field, D. Maskell, M. Kehoe, C. G. Dowson, N. Chanter, A. M. Whatmore, S. D. Bentley, and J. Parkhill. 2009. Evidence for niche adaptation in the genome of the bovine pathogen Streptococcus uberis. BMC Genomics 10:54. https://doi.org/ 10.1186/1471-2164-10-54.

Ward, W. R., J. W. Hughes, W. B. Faull, P. J. Cripps, J. P. Sutherland, and J. E. Sutherst. 2002. Observational study of temperature, moisture, $\mathrm{pH}$ and bacteria in straw bedding, and faecal consistency, cleanliness and mastitis in cows in four dairy herds. Vet. Rec. 151:199-206. https://doi.org/10.1136/vr.151.7.199.

Ye, J., G. Coulouris, I. Zaretskaya, I. Cutcutache, S. Rozen, and T. L. Madden. 2012. Primer-BLAST: A tool to design target-specific primers for polymerase chain reaction. BMC Bioinformatics 13:134. https://doi.org/10.1186/1471-2105-13-134.

Zadoks, R. N., L. L. Tikofsky, and K. J. Boor. 2005. Ribotyping of Streptococcus uberis from a dairy's environment, bovine feces and milk. Vet. Microbiol. 109:257-265. https://doi.org/10.1016/ j.vetmic.2005.05.008.

\section{ORCIDS}

Virginia E. Sherwin ( https://orcid.org/0000-0002-4604-9163

Martin J. Green () https://orcid.org/0000-0002-6408-6443

James A. Leigh (1 https://orcid.org/0000-0002-0307-2814

Sharon A. Egan ๑ https://orcid.org/0000-0001-6821-6690 